\title{
Signatures of Weyl Fermion Annihilation in a Correlated Kagome Magnet
}

\author{
Ilya Belopolski®, ${ }^{1,12,{ }^{*}}$ Tyler A. Cochran, ${ }^{1}$ Xiaoxiong Liu $\odot,{ }^{2}$ Zi-Jia Cheng, ${ }^{1}$ Xian P. Yang, ${ }^{1}$ Zurab Guguchia®, ${ }^{1,3}$ \\ Stepan S. Tsirkin $\odot,{ }^{2}$ Jia-Xin Yin, ${ }^{1}$ Praveen Vir, ${ }^{4}$ Gohil S. Thakur $\odot,{ }^{4,5}$ Songtian S. Zhang, ${ }^{1}$ Junyi Zhang, ${ }^{6}$ \\ Konstantine Kaznatcheev, ${ }^{7}$ Guangming Cheng, ${ }^{8}$ Guoqing Chang $\odot,{ }^{9}$ Daniel Multer, ${ }^{1}$ Nana Shumiya, ${ }^{1}$ \\ Maksim Litskevich, ${ }^{1}$ Elio Vescovo, ${ }^{7}$ Timur K. Kim $\odot,{ }^{10}$ Cephise Cacho $\odot,{ }^{10}$ Nan Yao, ${ }^{8}$ Claudia Felser $\odot,{ }^{4}$ \\ Titus Neupert๑, ${ }^{2}$ and M. Zahid Hasan ${ }^{1,8,11, \dagger}$ \\ ${ }^{1}$ Laboratory for Topological Quantum Matter and Spectroscopy (B7), Department of Physics, Princeton University, \\ Princeton, New Jersey 08544, USA \\ ${ }^{2}$ Department of Physics, University of Zurich, Winterthurerstrasse 190, 8057 Zurich, Switzerland \\ ${ }^{3}$ Laboratory for Muon Spin Spectroscopy, Paul Scherrer Institute, Villigen PSI, Switzerland \\ ${ }^{4}$ Max Planck Institute for Chemical Physics of Solids, Nöthnitzer Straße 40, 01187 Dresden, Germany \\ ${ }^{5}$ Faculty of Chemistry and Food Chemistry, Technische Universitat, 01069 Dresden, Germany \\ ${ }^{6}$ Department of Physics, Princeton University, Princeton, New Jersey 08544, USA \\ ${ }^{7}$ National Synchrotron Light Source II, Brookhaven National Laboratory, Upton, New York 11973, USA \\ ${ }^{8}$ Princeton Institute for Science and Technology of Materials, Princeton University, Princeton, New Jersey 08544, USA \\ ${ }^{9}$ Division of Physics and Applied Physics, School of Physical and Mathematical Sciences, Nanyang Technological University, \\ 21 Nanyang Link, 637371 Singapore, Singapore \\ ${ }^{10}$ Diamond Light Source, Didcot OX11 ODE, United Kingdom \\ ${ }^{11}$ Materials Sciences Division, Lawrence Berkeley National Laboratory, Berkeley, California 94720, USA \\ ${ }^{12}$ RIKEN Center for Emergent Matter Science (CEMS), Wako, Saitama 351-0198, Japan
}

(Received 2 September 2021; accepted 12 November 2021; published 17 December 2021)

\begin{abstract}
The manipulation of topological states in quantum matter is an essential pursuit of fundamental physics and next-generation quantum technology. Here we report the magnetic manipulation of Weyl fermions in the kagome spin-orbit semimetal $\mathrm{Co}_{3} \mathrm{Sn}_{2} \mathrm{~S}_{2}$, observed by high-resolution photoemission spectroscopy. We demonstrate the exchange collapse of spin-orbit-gapped ferromagnetic Weyl loops into paramagnetic Dirac loops under suppression of the magnetic order. We further observe that topological Fermi arcs disappear in the paramagnetic phase, suggesting the annihilation of exchange-split Weyl points. Our findings indicate that magnetic exchange collapse naturally drives Weyl fermion annihilation, opening new opportunities for engineering topology under correlated order parameters.
\end{abstract}

DOI: 10.1103/PhysRevLett.127.256403

Quantum magnets exhibiting electronic topology are attracting considerable interest for the magnetic manipulation of Weyl and Dirac quasiparticles, as well as their topological surface states [1-7]. To date, spectroscopic signatures of electronic topological ground states have been observed in several magnetic semimetals, comprising magnetic Weyl loops [8]; Weyl points [9-13]; and massive Dirac fermions [14]. In parallel, the magnetic manipulation of Weyl and Dirac fermions has been extensively explored in transport [15-20]. However, direct spectroscopic observation of magnetic control of topology remains challenging. Demonstrating coherent evolution of topological quasiparticles under varying magnetic order, such as the annihilation of Weyl points, offers the possibility to directly verify fundamental notions of topological band theory $[2,3,21,22]$. Furthermore, novel transport and optical effects are enabled by tuning the relative energies of Weyl loops and points [23-25], controlling their positions relative to the Fermi level $[8,26-29]$ and switching their topological surface states on and off [30,31].
We have investigated magnetic modulation of topological semimetallic states in a range of materials by spectroscopy, including $\mathrm{Fe}_{3} \mathrm{Sn}_{2}, \mathrm{Co}_{2} \mathrm{MnGa}$, PrAlGe, $\mathrm{Fe}_{3} \mathrm{GeTe}_{2}$, $\mathrm{TbMn}_{6} \mathrm{Sn}_{6}$, and $\mathrm{Co}_{3} \mathrm{Sn}_{2} \mathrm{~S}_{2}$ [8,13,32-34]. Some of these materials exhibit high magnetic transition temperatures $>600 \mathrm{~K}$, so that thermal broadening may fundamentally overwhelm magnetic evolution of the Weyl or Dirac state $[8,14]$. Other systems, such as PrAlGe, exhibit low transition temperatures of $\sim 10 \mathrm{~K}$, associated with only small magnetic perturbations to the electronic structure [13]. Even in materials such as $\mathrm{Fe}_{3} \mathrm{GeTe}_{2}$, with intermediate $T_{C}=230 \mathrm{~K}$, the thermal evolution appears to be dominated by a suppression of quasiparticle lifetime, without significant coherent evolution of the dispersion [35-37]. Using newly available high-quality single crystals combined with state-of-the-art variable-temperature photoemission spectroscopy, we have found that a large and previously overlooked energy shift of a topological spin-orbit gapped Weyl loop occurs in $\mathrm{Co}_{3} \mathrm{Sn}_{2} \mathrm{~S}_{2}$ across 
$T_{C}=176 \mathrm{~K}$ [38-43]. This shift takes place together with a magnetic exchange gap collapse that suggests a ferromagnetic Weyl to paramagnetic Dirac loop transition on raising temperature. This transition is further accompanied by the removal of candidate topological Fermi arc surface states and the annihilation of Weyl points.

Materials with inversion symmetry, mirror symmetry, and ferromagnetism provide a unique platform for a magnetic-topological phase transition. The ferromagnetism produces singly degenerate spin-split bands. In the limit of weak spin-orbit coupling (SOC), mirror symmetry can then give rise to Weyl loops on mirror planes of the bulk Brillouin zone [8,44-46]. A Weyl loop is a closed curve along which the bands are twofold degenerate everywhere; it is characterized by a $\pi$ Berry phase topological invariant and a linear energy-momentum dispersion everywhere along the loop. If the magnetic order is removed and no spin splitting remains, opposite-spin partner Weyl loops naturally collapse into a Dirac loop, a closed curve along which the bands are fourfold degenerate everywhere [46-48]. Weyl loops under SOC typically gap out, concentrating a loop of Berry curvature in momentum space, leading to a giant anomalous Hall response $[8,10]$, large anomalous Nernst effect [29,49], large optical Hall conductivity [40], and other exotic response. Under SOC, Weyl loops may also leave behind some discrete number of Weyl points. By contrast, Dirac points are generically unstable under inversion and time-reversal symmetry [50], so that Dirac loops under SOC gap out fully. As a result, in this scenario upon magnetic exchange collapse the Weyl points generically annihilate.

$\mathrm{Co}_{3} \mathrm{Sn}_{2} \mathrm{~S}_{2}$ crystallizes in space group $R \overline{3} 2 / m$ (No. 166), with dihedral point group $D_{3 d}$, which includes inversion symmetry and three mirror planes [Fig. 1(a), S3]. The system is ferromagnetic, with Curie temperature $T_{C}=$ $176 \mathrm{~K}$ [51,52]. Keeping in mind the mirror symmetry and ferromagnetic order, we explore our $\mathrm{Co}_{3} \mathrm{Sn}_{2} \mathrm{~S}_{2}$ samples by ARPES at $20 \mathrm{~K}$. Measuring with incident photon energy (a)
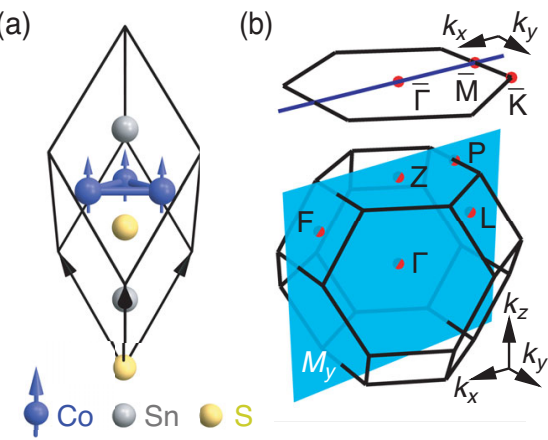

(c)

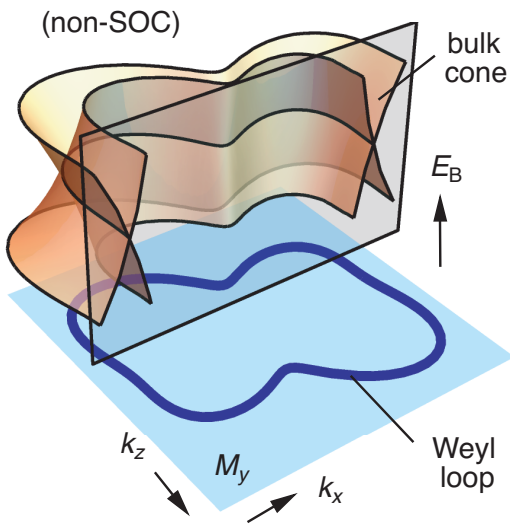

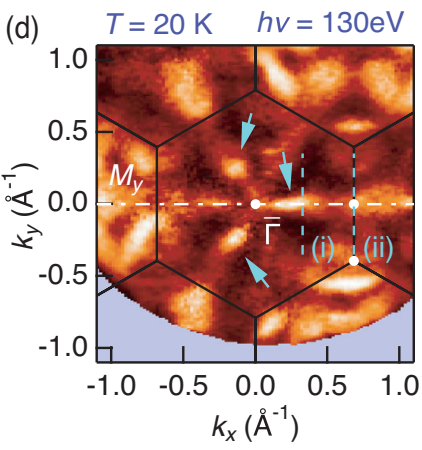
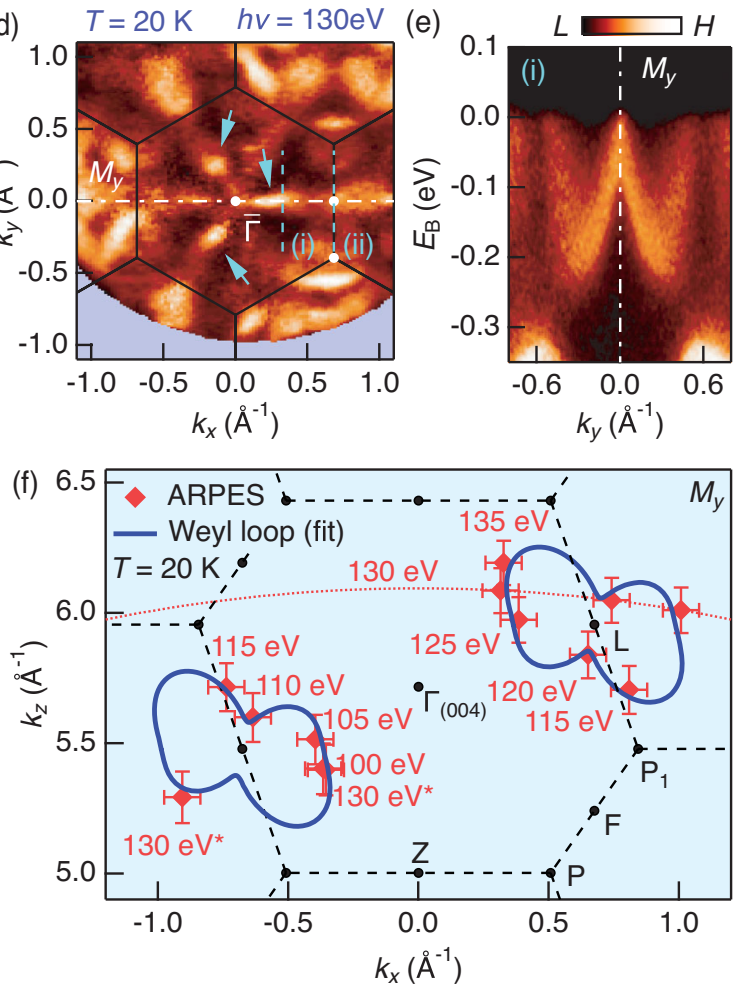

FIG. 1. Topological magnetic Weyl loop. (a) Primitive unit cell of ferromagnetic $\mathrm{Co}_{3} \mathrm{Sn}_{2} \mathrm{~S}_{2}$, with mirror symmetry. (b) Bulk and (001) surface Brillouin zones with bulk mirror plane $\left(M_{y}\right.$, cyan) and several high-symmetry points (red). (c) In the absence of spin-orbit coupling (SOC), the combination of mirror symmetry and ferromagnetism generically gives rise to Weyl loops, which live in the mirror planes of the bulk Brillouin zone. A Weyl loop exhibits a ring of band crossings along a closed curve in momentum space (blue loop) with a linear cone dispersion on any energy-momentum slice through the loop. Under SOC, the Weyl loop typically gaps, possibly leaving behind Weyl points. (d) ARPES Fermi surface at $T=20 \mathrm{~K}$ and photon energy $h \nu=130$ eV, exhibiting multiple dot features (cyan arrows) on the mirror planes $(\bar{\Gamma}-\bar{M})$. (e) Cone dispersion at the Fermi level on an energy-momentum spectrum through the dot feature (Cut (i)). (f) Collecting cone dispersions for a range of $h \nu$ suggests a loop of band crossings (red diamonds) living in $M_{y}$ and encircling the bulk $L$ point (Figs. S4, S5). Different $h \nu$ sample different out-of-plane $k_{z}$ momenta; representative example shown for $130 \mathrm{eV}$ (dotted red curve). The crossing points can be fit by a low-order polar coordinate Fourier decomposition around the $L$ point (blue curve [53]), mapping out the trajectory of the Weyl loop. 
$h \nu=130 \mathrm{eV}$, we observe pointlike electronic structures on $M_{y}$ [cyan arrows, Fig. 1(d); the mirror planes correspond to $\bar{\Gamma}-\bar{M}]$. On cuts along $k_{y}$ through the pointlike features, we observe cone dispersions straddling the mirror plane $M_{y}$ [Fig. 1(e)]. On an energy-momentum cut along $k_{x}$, within the mirror plane, we again observe conelike dispersions (Fig. S4). The observation of cone dispersions along both $k_{x}$ and $k_{y}$, coming together at point Fermi surfaces, suggests a set of band crossings living in the momentum-space mirror plane. To systematically understand the evolution of the band crossings along the out-of-plane momentum-space direction $k_{z}$ we acquire analogous datasets at a range of photon energies, from $h \nu=100$ to $135 \mathrm{eV}$ (Fig. S5). We find that the cones persist in $h \nu$, with crossing points consistently on the $M_{y}$ plane, but at varying $\left(k_{x}, k_{z}\right)$ coordinates [red diamonds, Fig. 1(f)]. Taken together, these crossing points appear to form an extended nodal electronic state encircling the $L$ point of the bulk Brillouin zone, suggesting the observation of a bulk loop node in $\mathrm{Co}_{3} \mathrm{Sn}_{2} \mathrm{~S}_{2}$. Since the system is ferromagnetic with generically singly degenerate bands, we interpret this loop node as a Weyl loop [Fig. 1(c)]. To extract the complete trajectory of the loop, we fit the ARPES locations of the cone dispersions to a low-order polar Fourier decomposition around the $L$ point of the bulk Brillouin zone [blue loop, Fig. 1(f); see Supplemental Material [53] for fitting parameter values]. In this way we extract the full momentum-space trajectory of the Weyl loop from photoemission data alone.

Next we explore the evolution of the Weyl loop with temperature, focusing on Cut (i). We systematically cycle the temperature of our samples from $20 \mathrm{~K}$ to $290 \mathrm{~K}$ and back to $20 \mathrm{~K}$, moving across $T_{C}=176 \mathrm{~K}$. On raising the temperature, we observe a dramatic evolution of the Weyl cone on a large energy scale of $\sim 0.1 \mathrm{eV}$ [Figs. 2(a) and 2(b); S8], with the cone appearing to recede above $E_{F}$. We next assemble the momentum distribution curves (MDCs) of Cut (i) at $E_{F}$ for all temperatures [Fig. 2(c)]. Upon cycling the temperature, we observe a prominent and reversible evolution of the Weyl cone across $T_{C}$, consistent with a magnetic phase transition. For further insight, we examine additional spectra on Cut (ii), obtained during the course of the same measurement, and we consider a set of deep bands $\sim 0.3 \mathrm{eV}$ below $E_{F}$, which are predominantly formed from the same exchange-split Co $3 d a_{1 g}$ and $e_{g}$ manifolds as the Weyl loop [Figs. 2(d); S11]. At $20 \mathrm{~K}$, these
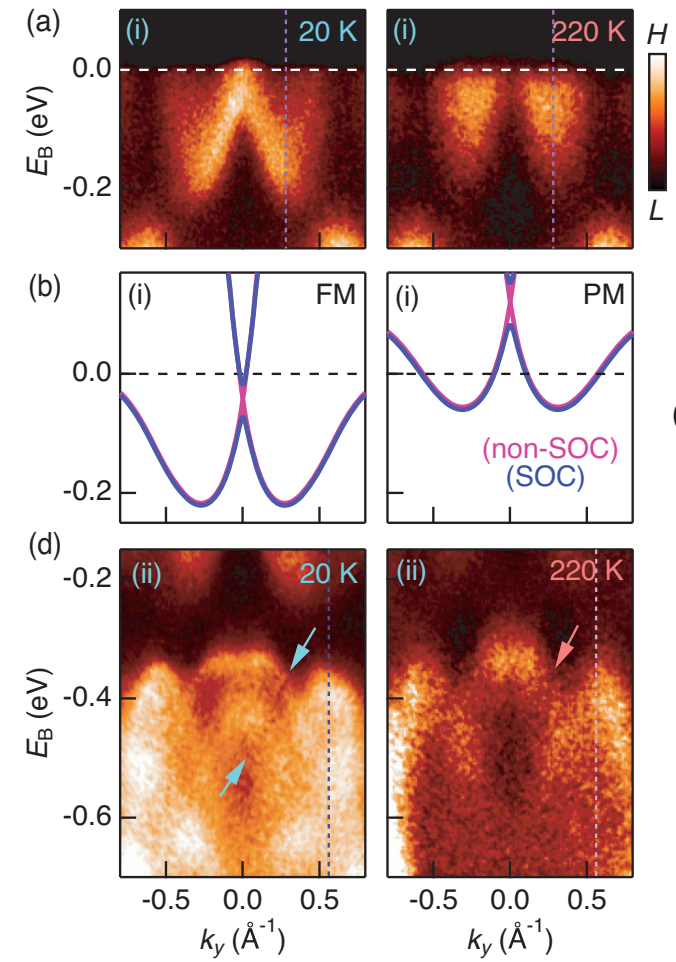

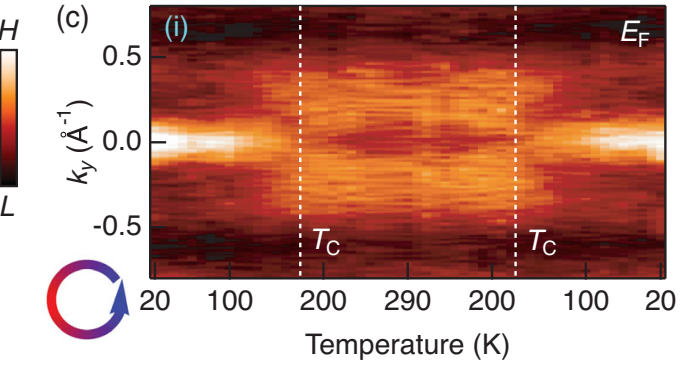

(e)
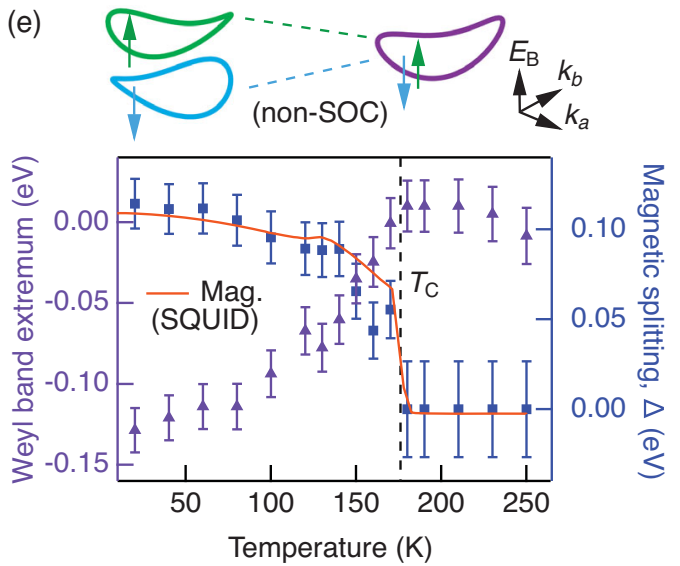

FIG. 2. Magnetic Weyl to Dirac loop collapse. (a) Cut (i) at $20 \mathrm{~K}$ and $220 \mathrm{~K}$, with (b) corresponding ab initio calculation. Left: calculation in the ferromagnetic state through the Weyl loop, without SOC (magenta) and with SOC (blue). Right: calculation in the nonmagnetic state through the Dirac loop. (c) Momentum distribution curves (MDCs) of Cut (i) at the Fermi level for the full temperature cycle, $20 \mathrm{~K} \rightarrow 290 \mathrm{~K} \rightarrow 20 \mathrm{~K}$. (d) Cut (ii), defined in Fig. 1(d), exhibiting clear splittings in deeper energy bands at $20 \mathrm{~K}$ (left), which collapse at $220 \mathrm{~K}$ (right). (e) Energy extremum of the Weyl loop band, extracted from the temperature dependence on Cut (i), obtained by Lorentzian fitting of energy distribution curves [EDCs, dotted lines in (a)]. Also, the magnetic exchange splitting as a function of temperature, obtained from Cut (ii) by Lorentzian fitting to EDCs [dotted lines in (d)] and compared with the magnetization $M(T)$. Cartoon: exchange gap collapse of two opposite-spin Weyl loop partners (blue and green) into a single Dirac loop (purple). 
deep valence bands exhibit clear splitting, consistent with the material's ferromagnetic order. Upon raising the temperature, the splitting appears to vanish and these deep bands collapse together, suggesting a paramagnetic state with spin-degenerate bands. By examining the evolution of the deep bands, we circumvent the limitations of the photoemission $E_{F}$ cutoff and observe direct signatures of a prominent magnetic exchange gap collapse across $T_{C}$ in $\mathrm{Co}_{3} \mathrm{Sn}_{2} \mathrm{~S}_{2}$.

To relate the Weyl loop temperature evolution to the magnetic exchange gap collapse, we consider more carefully the interplay between topology and ferromagnetism. In $a b$ initio calculation, in the absence of SOC and in the ferromagnetic state, the Weyl loop arises as a crossing of two spin-majority bands, with a spin-minority partner Weyl loop above the Fermi level [schematic blue and green loops, Figs. 2(e); S6]. In a nonmagnetic ab initio calculation, the exchange gap vanishes and these two Weyl loops coincide, forming a spinless loop crossing - a Dirac loop (purple loop). Comparing the $a b$ initio calculations with ARPES, we find that the magnetic Weyl and nonmagnetic Dirac nodes exhibit overall agreement with the ferromagnetic and paramagnetic spectra, respectively [magenta traces, Fig. 2(b)]. Note that including SOC in our $a b$ initio results does not alter this interpretation, although the expected gap appears in both loop nodes (blue traces). The observation that the loop recedes above $E_{F}$ on increasing temperature is also consistent with maintenance of charge balance in the spin-degenerate electronic structure, further indicating a paramagnetic Dirac loop. We further reduced the magnetic moment in our samples via nickel (Ni) doping, and again observed a persistent loop node electronic structure despite suppression of the ferromagnetism (Fig. S1). Taken together, our systematic ARPES spectra and $a b$ initio calculations suggest that we have observed the collapse of two opposite-spin ferromagnetic Weyl loops into a paramagnetic Dirac loop.

To quantitatively characterize the Weyl loop collapse with temperature, we perform a Lorentzian fit of energy distribution curves (EDCs) through the extremum of the Weyl loop band [Cut (i), dotted line, Fig. 2(a)]. The extracted Weyl band extremum exhibits a clear evolution upward in $E_{\mathrm{B}}$ as the temperature increases, $20 \mathrm{~K} \rightarrow 250 \mathrm{~K}$, consistent with exchange gap collapse [Fig. 2(e)]. We further extract the exchange gap $\Delta(T)$ on EDCs through the deep bands [Cut (ii), dotted line, Fig. 2(d)] and compared the resulting $\Delta(T)$ with the magnetization $M(T)$ as measured by a SQUID. For $T<T_{C}$ we find that the exchange splitting tracks $M(T)$. For $T>T_{C}$ we no longer observe an exchange splitting within our spectral linewidth, consistent with the absence of magnetization. Remarkably, the observed exchange gap and Weyl band shift are both $\sim 0.12 \mathrm{eV}$, suggesting a complete collapse of the opposite-spin partner Weyl loops across $T_{C}$ and the formation of a spin-degenerate paramagnetic Dirac loop.
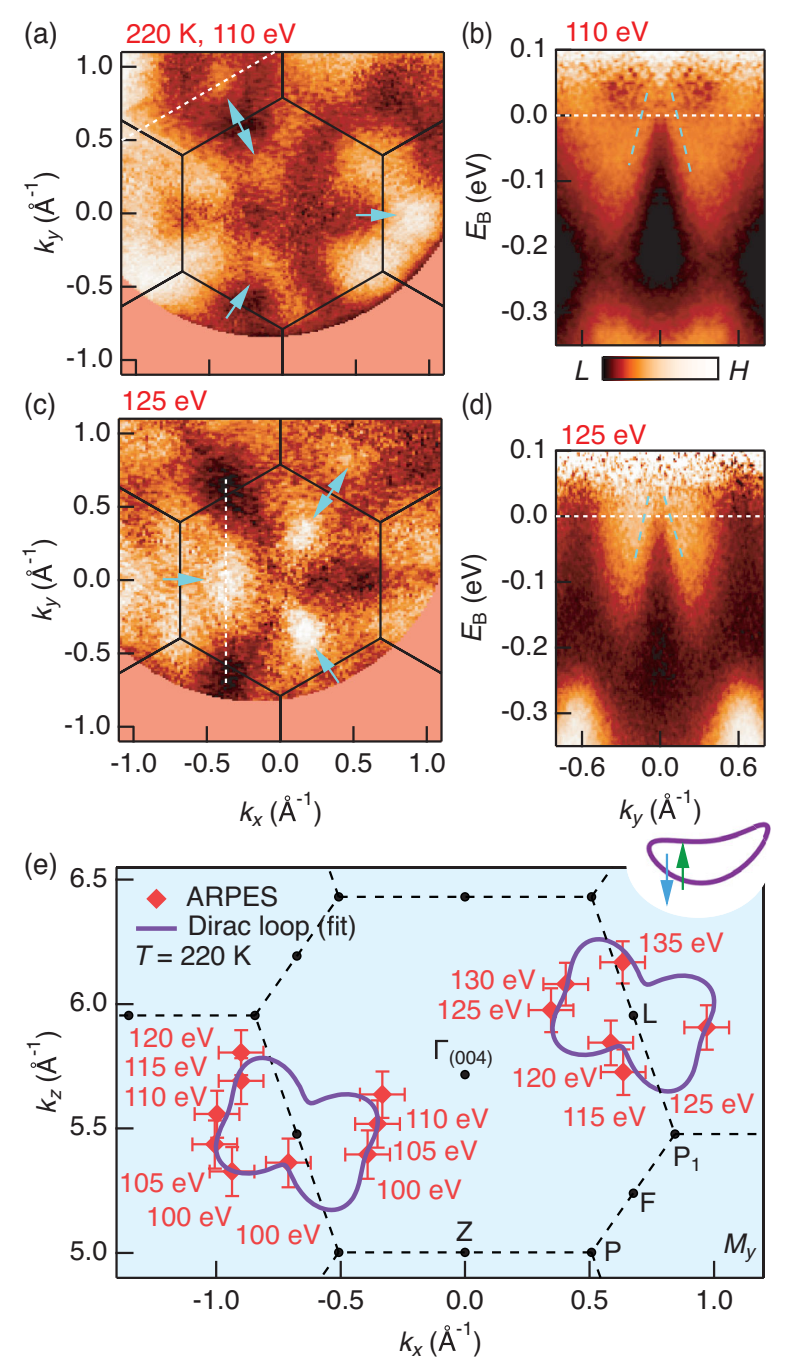

FIG. 3. Paramagnetic Dirac loop. (a) ARPES isoenergy contour slightly above $E_{F}$, at $h \nu=110 \mathrm{eV}$, acquired at $220 \mathrm{~K}$, exhibiting pointlike features (cyan arrows) along $\bar{\Gamma}-\bar{M}$ (corresponding to $M_{y}$ and the symmetry-related mirror planes). (b) Energy-momentum cut through the pointlike feature, exhibiting conelike spectral weight [cut location: white line in (a)]. (c),(d) Analogous to (a), (b), at $125 \mathrm{eV}$. (e) Locations of cones observed for all $h \nu$ (red diamonds, Fig. S7). Cones on symmetry-related mirror planes are plotted all together in a single momentum-space mirror plane $M_{y}$. Data points fit to a low-order polar coordinate Fourier decomposition around $L$ (purple curves), mapping out the trajectory of the Dirac loop.

To further explore the paramagnetic Dirac loop we park our apparatus at $220 \mathrm{~K}$, well into the paramagnetic phase. At a range of $h \nu$ we observe characteristic pointlike isoenergy contours on $M_{y}$ and related mirror planes [Figs. 3(a) and 3(c); S7]. Energy-momentum spectra through these pointlike contours further exhibit conelike spectral weight straddling $M_{y}$, indicative of Dirac loop cone dispersions above $E_{F}$ [Figs. 3(b) and 3(d)]. The presence of multiple cone features straddling $M_{y}$ at a range of $h \nu$ again suggests an extended nodal electronic structure 

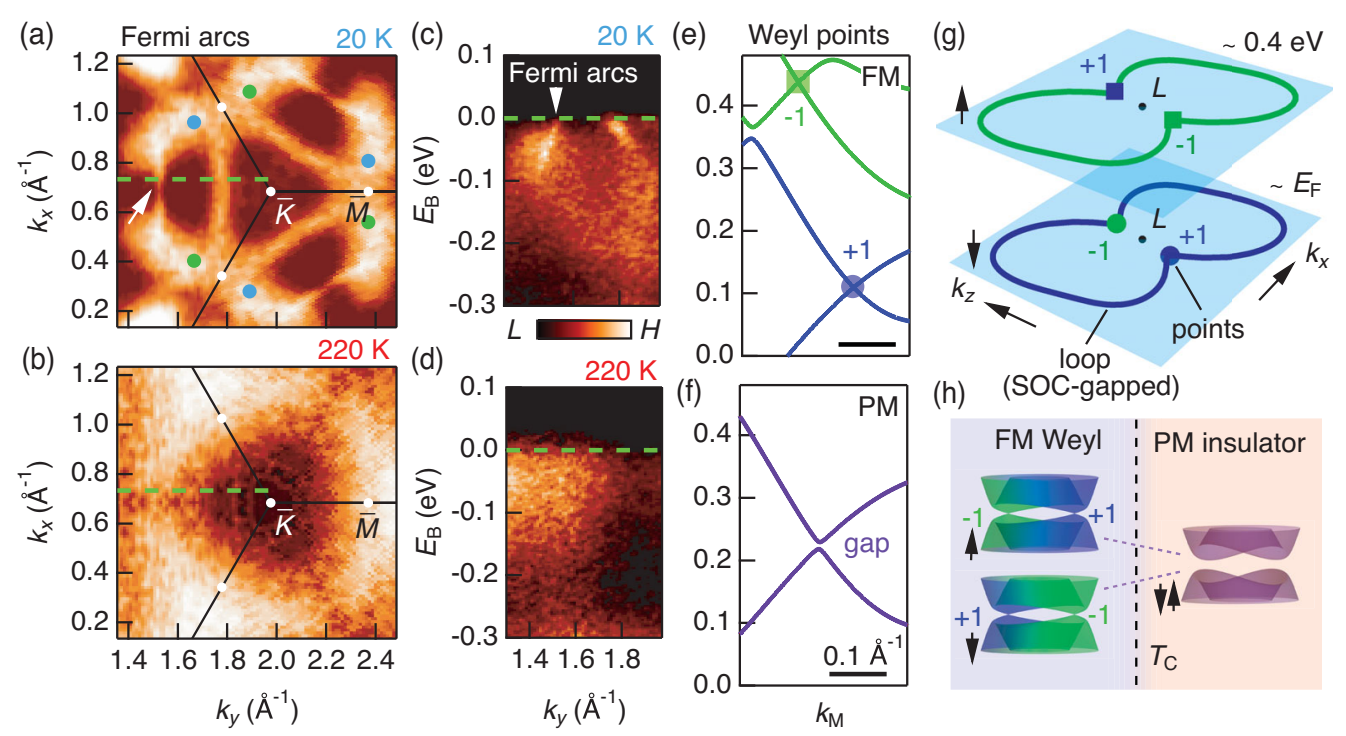

FIG. 4. Evidence for Fermi arc and Weyl point annihilation. (a) Fermi surface acquired at $T=20 \mathrm{~K}, h \nu=130 \mathrm{eV}$, exhibiting candidate Fermi arc surface states (white arrow) near the Weyl point locations, as predicted by ab initio calculations (blue, green circle; Fig. S9). Data symmetrized for clarity. (b) Analogous Fermi surface at 220 K, with no signature of Fermi arcs. (c) Energy-momentum cut through the candidate Fermi arcs at $20 \mathrm{~K}$ [green dotted line, (a)]. (d) Analogous cut at $220 \mathrm{~K}$. (e) Calculation in the ferromagnetic state, in the presence of SOC, slicing through a pair of exchange-split Weyl points of opposite chirality. (f) Analogous calculation, nonmagnetic state. The two partner Weyl points annihilate, opening a gap of $12 \mathrm{meV}$. (g) Schematic of the ferromagnetic Weyl loop (non-SOC) and Weyl point (SOC) configuration. (h) Schematic phase diagram: spin-orbit gapped ferromagnetic Weyl loops collapse to a paramagnetic Dirac loop across $T_{C}$. Concurrently, the exchange-split Weyl points annihilate.

confined to the mirror plane. Since we are in the paramagnetic phase with generically spin-degenerate bands, we interpret these candidate band crossings as fourfold degenerate, forming a Dirac loop. By analogy with our analysis in the ferromagnetic phase, we again systematically collect the locations of all cone features observed in the paramagnetic phase for $h \nu$ from 100 to $135 \mathrm{eV}$ and experimentally extract the full momentum-space trajectory of the Dirac loop [red diamonds, Fig. 3(e)]. Ab initio calculations of the Weyl and Dirac loops in the ferromagnetic and nonmagnetic states also agree with the experimentally observed trajectories (Fig. S2). A loop node electronic structure persisting into the paramagnetic phase of $\mathrm{Co}_{3} \mathrm{Sn}_{2} \mathrm{~S}_{2}$ again suggests the observation of a ferromagnetic Weyl to paramagnetic Dirac loop collapse.

We next consider the fine structure of the Weyl loop collapse associated with SOC. In $\mathrm{Co}_{3} \mathrm{Sn}_{2} \mathrm{~S}_{2}$, ab initio calculations along with ARPES and STM investigations suggest that each Weyl loop under SOC produces two Weyl points above the Fermi level, with signatures of topological Fermi arc surface states extending below the Fermi level. [9-12,38-41]. Our observation of a Weyl to Dirac loop transition naturally motivates investigation of Fermi arc and Weyl point annihilation across $T_{C}$. At $20 \mathrm{~K}$, we observe sharp arc-shaped states near the expected Weyl points, consistent with topological Fermi arcs in ab initio calculation [Figs. 4(a) and 4(c); S9]. At $220 \mathrm{~K}$ these Fermi arcs vanish, leaving behind bulk pockets broadly consistent with the low temperature spectra [Figs. 4(b) and 4(d)]. The disappearance of the Fermi arcs above $T_{C}$ provides evidence for the annihilation of Weyl points in the paramagnetic phase. To further characterize this annihilation, we consider the $a b$ initio band structure under ferromagnetic order on a momentum-space path connecting a pair of exchange-split Weyl points [Fig. 4(e)]. Upon exchange collapse, these partner Weyl points come together and annihilate, opening a gap [Fig. 4(f)].

Our systematic variable-temperature ARPES experiments suggest that pairs of ferromagnetic Weyl loops collapse into paramagnetic Dirac loops across $T_{C}$ in $\mathrm{Co}_{3} \mathrm{Sn}_{2} \mathrm{~S}_{2}$ [Figs. 4(g) and 4(h)]. Taken together with $a b$ initio calculations, our results additionally provide evidence for the annihilation of Fermi arcs and Weyl points concomitant with this transition. Our findings suggest a general mechanism for Weyl fermion annihilation, where the annihilation is driven by magnetic exchange gap collapse and takes place predominantly along the energy axis, rather than in momentum $[3,21,62]$. This novel mechanism should occur naturally in many quantum magnets and motivates exploration of the rich topological evolution associated with the onset of magnetic order, including novel magnetic topological insulators. Such interplay between magnetism and topology may also pave the way to magnetic design of correlated topological states with exotic transport and optical response.

The authors thank D. Lu and M. Hashimoto at Beamline 5-2 of the Stanford Synchrotron Radiation Lightsource 
(SSRL) at the SLAC National Accelerator Laboratory, CA, USA for support. The authors thank Diamond Light Source for access to Beamline I05 (SI17924, SI19313). This research used Beamline 21-ID-1 (ESM-ARPES) of the National Synchrotron Light Source II, a U.S. Department of Energy (DOE) Office of Science User Facility operated for the DOE Office of Science by Brookhaven National Laboratory under Contract No. DE-SC0012704. The authors also acknowledge use of Princeton University's Imaging and Analysis Center, which is partially supported by the Princeton Center for Complex Materials (PCCM), a National Science Foundation (NSF)-MRSEC program (DMR-2011750). Use of the Stanford Synchrotron Radiation Lightsource (SSRL), SLAC National Accelerator Laboratory, is supported by the U.S. Department of Energy, Office of Science, Office of Basic Energy Sciences, under Contract No. DE-AC02-76SF00515. T. A. C. acknowledges the support of the National Science Foundation Graduate Research Fellowship Program (DGE-1656466). T. N. and S. S. T. acknowledge support from the European Union Horizon 2020 Research and Innovation Program (ERC-StG-Neupert-757867-PARATOP). S. S. T. also acknowledge support from the Swiss National Science Foundation (Grant No. PP00P2-176877). X. L. acknowledges financial support from the China Scholarship Council. G. C. would like to acknowledge the support of the National Research Foundation, Singapore under its NRF Fellowship Award (NRF-NRFF13-2021-0010) and the Nanyang Assistant Professorship grant from Nanyang Technological University. M.Z. H. acknowledges visiting scientist support at Berkeley Lab (LBNL) during the early phases of this work. Work at Princeton University was supported by the Gordon and Betty Moore Foundation (Grants No. GBMF4547 and No. GBMF9461; M. Z. H.). The ARPES and theoretical work were supported by the United States Department of Energy (US DOE) under the Basic Energy Sciences programme (Grant No. DOE/BES DE-FG-02-05ER46200; M.Z.H.). G. S. T. thanks the Würzburg-Dresden Cluster of Excellence on Complexity and Topology in Quantum Matter - ct.qmat (EXC 2147) for postdoctoral funding. C. F. acknowledges the DFG through SFB 1143 (project ID. 247310070) and the WürzburgDresden Cluster of Excellence on Complexity and Topology in Quantum Matter ct.qmat (EXC2147, project ID. 39085490).

I. B., T. A. C., X. L., and Z.-J. C. contributed equally to this work.

*ilya.belopolski@riken.jp †mzhasan@princeton.edu

[1] E. H. da Silva Neto, "Weyl"ing away time-reversal symmetry, Science 365, 1248 (2019).

[2] N. P. Armitage, E. J. Mele, and A. Vishwanath, Weyl and Dirac semimetals in three-dimensional solids, Rev. Mod. Phys. 90, 015001 (2018).
[3] M.Z. Hasan, S.-Y. Xu, I. Belopolski, and S.-M. Huang, Discovery of Weyl fermion semimetals and topological Fermi arc states, Annu. Rev. Condens. Matter Phys. 8, 289 (2017).

[4] J. Maciejko and G. A. Fiete, Fractionalized topological insulators, Nat. Phys. 11, 385 (2015).

[5] M.Z. Hasan et al., Weyl, Dirac and high-fold chiral fermions in topological quantum matter, Nat. Rev. Mater. 6, 784 (2021).

[6] B. Keimer and J.E. Moore, The physics of quantum materials, Nat. Phys. 13, 1045 (2017).

[7] D. N. Basov, R. D. Averitt, and D. Hsieh, Towards properties on demand in quantum materials, Nat. Mater. 16, 1077 (2017).

[8] I. Belopolski et al., Discovery of topological Weyl fermion lines and drumhead surface states in a room temperature magnet, Science 365, 1278 (2019).

[9] Q. Wang, Y. Xu, R. Lou, Z. Liu, M. Li, Y. Huang, D. Shen, H. Weng, S. Wang, and H. Lei, Large intrinsic anomalous Hall effect in half-metallic ferromagnet $\mathrm{Co}_{3} \mathrm{Sn}_{2} \mathrm{~S}_{2}$ with magnetic Weyl fermions, Nat. Commun. 9, 3681 (2018).

[10] E. Liu et al., Giant anomalous Hall effect in a ferromagnetic kagome-lattice semimetal, Nat. Phys. 14, 1125 (2018).

[11] N. Morali, R. Batabyal, P. Kumar Nag, E. Liu, Q. Xu, Y. Sun, B. Yan, C. Felser, N. Avraham, and H. Beidenkopf, Fermi-arc diversity on surface terminations of the magnetic Weyl semimetal $\mathrm{Co}_{3} \mathrm{Sn}_{2} \mathrm{~S}_{2}$, Science 365, 1286 (2019).

[12] D.F. Liu et al., Magnetic Weyl semimetal phase in a Kagomé crystal, Science 365, 1282 (2019).

[13] D. S. Sanchez et al., Observation of Weyl fermions in a magnetic non-centrosymmetric crystal, Nat. Commun. 11, 3356 (2020).

[14] L. Ye et al., Massive Dirac fermions in a ferromagnetic kagome metal, Nature (London) 555, 638 (2018).

[15] J. Hu, S.-Y. Xu, N. Ni, and Z. Mao, Transport of topological semimetals, Annu. Rev. Condens. Matter Phys. 49, 207 (2019).

[16] J. Xiong, S. K. Kushwaha, T. Liang, J. W. Krizan, M. Hirschberger, W. Wang, R. J. Cava, and N. P. Ong, Evidence for the chiral anomaly in the Dirac semimetal $\mathrm{Na}_{3} \mathrm{Bi}$, Science 350, 413 (2015).

[17] C.-L. Zhang et al., Magnetic-tunnelling-induced Weyl node annihilation in TaP, Nat. Phys. 13, 979 (2017).

[18] K. Manna et al., From Colossal to Zero: Controlling the Anomalous Hall Effect in Magnetic Heusler Compounds via Berry Curvature Design, Phys. Rev. X 8, 041045 (2018).

[19] A. Sakai et al., Giant anomalous Nernst effect and quantumcritical scaling in a ferromagnetic semimetal, Nat. Phys. 14, 1119 (2018).

[20] K. Kuroda et al., Evidence for magnetic Weyl fermions in a correlated metal, Nat. Mater. 16, 1090 (2017).

[21] A. A. Burkov and L. Balents, Weyl Semimetal in a Topological Insulator Multilayer, Phys. Rev. Lett. 107, 127205 (2011).

[22] X. Wan, A. M. Turner, A. Vishwanath, and S. Y. Savrasov, Topological semimetal and Fermi-arc surface states in the electronic structure of pyrochlore iridates, Phys. Rev. B 83, 205101 (2011).

[23] A. A. Zyuzin, S. Wu, and A. A. Burkov, Weyl semimetal with broken time reversal and inversion symmetries, Phys. Rev. B 85, 165110 (2012). 
[24] S. F. Islam and A. A. Zyuzin, Collective modes in imbalanced nodal line semimetal, arXiv:2108.13951.

[25] S. Zhong, J. E. Moore, and I. Souza, Gyrotropic Magnetic Effect and the Magnetic Moment on the Fermi Surface, Phys. Rev. Lett. 116, 077201 (2016).

[26] Q. Ma et al., Direct optical detection of Weyl fermion chirality in a topological semimetal, Nat. Phys. 13, 842 (2017).

[27] G. B. Osterhoudt et al., Colossal mid-infrared bulk photovoltaic effect in a type-I weyl semimetal, Nat. Mater. 18, 471 (2019).

[28] S. S.-L. Zhang, A. A. Burkov, I. Martin, and O. G. Heinonen, Spin-to-Charge Conversion in Magnetic Weyl Semimetals, Phys. Rev. Lett. 123, 187201 (2019).

[29] A. Sakai et al., Iron-based binary ferromagnets for transverse thermoelectric conversion, Nature (London) 581, 53 (2020).

[30] J. C. W. Song and M. S. Rudner, Fermi arc plasmons in Weyl semimetals, Phys. Rev. B 96, 205443 (2017).

[31] F. Adinehvand, Z. Faraei, T. Farajollahpour, and S. A. Jafari, Sound of Fermi arcs: a linearly dispersing gapless surface plasmon mode in undoped Weyl semimetals, Phys. Rev. B 100, 195408 (2019).

[32] J.-X. Yin et al., Giant and anisotropic many-body spin-orbit tunability in a strongly correlated kagome magnet, Nature (London) 562, 91 (2018).

[33] J.-X. Yin et al., Quantum-limit Chern topological magnetism in $\mathrm{TbMn}_{6} \mathrm{Sn}_{6}$, Nature (London) 583, 533 (2020).

[34] J.-X. Yin et al., Negative flat band magnetism in a spinorbit-coupled correlated kagome magnet, Nat. Phys. 15, 443 (2019).

[35] K. Kim et al., Large anomalous Hall current induced by topological nodal lines in a ferromagnetic van der Waals semimetal, Nat. Mater. 17, 794 (2018).

[36] Y. Zhang et al., Emergence of Kondo lattice behavior in a van der Waals itinerant ferromagnet, $\mathrm{Fe}_{3} \mathrm{GeTe}_{2}$, Sci. Adv. 4, eaao6791 (2018).

[37] X. Xu et al., Signature for non-Stoner ferromagnetism in the van der Waals ferromagnet $\mathrm{Fe}_{3} \mathrm{GeTe}_{2}$, Phys. Rev. B 101, 201104(R) (2020).

[38] Q. Xu, E. Liu, W. Shi, L. Muechler, J. Gayles, C. Felser, and Y. Sun, Topological surface Fermi arcs in the magnetic Weyl semimetal $\mathrm{Co}_{3} \mathrm{Sn}_{2} \mathrm{~S}_{2}$, Phys. Rev. B 97, 235416 (2018).

[39] Z. Guguchia et al., Tunable anomalous Hall conductivity through volume-wise magnetic competition in a topological kagome magnet, Nat. Commun. 11, 559 (2020).

[40] Y. Okamura et al., Giant magneto-optical responses in magnetic Weyl semimetal $\mathrm{Co}_{3} \mathrm{Sn}_{2} \mathrm{~S}_{2}$, Nat. Commun. 11, 4619 (2020).

[41] R. Yang, T. Zhang, L. Zhou, Y. Dai, Z. Liao, H. Weng, and $X$. Qiu, Magnetization-Induced Band Shift in Ferromagnetic Weyl Semimetal $\mathrm{Co}_{3} \mathrm{Sn}_{2} \mathrm{~S}_{2}$, Phys. Rev. Lett. 124, 077403 (2020).

[42] A. Rossi et al., Electronic structure and topology across $T_{\mathrm{C}}$ in magnetic Weyl semimetal $\mathrm{Co}_{3} \mathrm{Sn}_{2} \mathrm{~S}_{2}$, arXiv:2105.08265.

[43] D. F. Liu et al., Topological phase transition in a magnetic Weyl semimetal, arXiv:2106.03229.

[44] Y. Wang and R. Nandkishore, Topological surface superconductivity in doped Weyl loop materials, Phys. Rev. B 95, 060506(R) (2017).
[45] O. Stenull, C. L. Kane, and T. C. Lubensky, Topological Phonons and Weyl Lines in Three Dimensions, Phys. Rev. Lett. 117, 068001 (2016).

[46] R. Nandkishore, Weyl and Dirac loop superconductors, Phys. Rev. B 93, 020506(R) (2016).

[47] Y. Kim, B. J. Wieder, C. L. Kane, and A. M. Rappe, Dirac Line Nodes in Inversion-Symmetric Crystals, Phys. Rev. Lett. 115, 036806 (2015).

[48] A. Yamakage, Y. Yamakawa, Y. Tanaka, and Y. Okamoto, Line-node Dirac semimetal and topological insulating phase in noncentrosymmetric pnictides $\operatorname{CaAg} X(X=\mathrm{P}$, As), J. Phys. Soc. Jpn. 85, 013708 (2016).

[49] S. N. Guin et al., Zero-field nernst effect in a ferromagnetic kagome-lattice Weyl-semimetal $\mathrm{Co}_{3} \mathrm{Sn}_{2} \mathrm{~S}_{2}$, Adv. Mater. 31, 1806622 (2019).

[50] S. Murakami, Phase transition between the quantum spin Hall and insulator phases in 3D: Emergence of a topological gapless phase, New J. Phys. 9, 356 (2007).

[51] P. Vaqueiro and G. G. Sobany, A powder neutron diffraction study of the metallic ferromagnet $\mathrm{Co}_{3} \mathrm{Sn}_{2} \mathrm{~S}_{2}$, Solid State Sci. 11, 513 (2009).

[52] W. Schnelle, A. Leithe-Jasper, H. Rosner, F. M. Schappacher, R. Pöttgen, F. Pielnhofer, and R. Weihrich, Ferromagnetic ordering and half-metallic state of $\mathrm{Sn}_{2} \mathrm{Co}_{3} \mathrm{~S}_{2}$ with the shandite-type structure, Phys. Rev. B 88, 144404 (2013).

[53] See Supplemental Material at http://link.aps.org/ supplemental/10.1103/PhysRevLett.127.256403 for methods and additional systematics, which including Refs. [54-61].

[54] G. S. Thakur, P. Vir, S. N. Guin, C. Shekhar, R. Weihrich, Y. Sun, N. Kumar, and C. Felser, Intrinsic anomalous hall effect in Ni-substituted magnetic Weyl semimetal $\mathrm{Co}_{3} \mathrm{Sn}_{2} \mathrm{~S}_{2}$, Chem. Mater. 32, 1612 (2020).

[55] M. Hoesch et al., A facility for the analysis of the electronic structures of solids and their surfaces by synchrotron radiation photoelectron spectroscopy, Rev. Sci. Instrum. 88, 013106 (2017).

[56] G. Kresse and J. Furthmueller, Efficient iterative schemes for $a b$ initio total-energy calculations using a plane-wave basis set, Phys. Rev. B 54, 11169 (1996).

[57] G. Kresse and J. Furthmueller, Efficiency of ab-initio total energy calculations for metals and semiconductors using a plane-wave basis set, Comput. Mater. Sci. 6, 15 (1996).

[58] J. P. Perdew, K. Burke, and M. Ernzerhof, Generalized Gradient Approximation Made Simple, Phys. Rev. Lett. 77, 3865 (1996).

[59] Q. S. Wu, S. Zhang, H.-F. Song, M. Troyer, and A. A. Soluyanov, WannierTools: An open-source software package for novel topological materials, Comput. Phys. Commun. 224, 405 (2018).

[60] G. Pizzi et al., Wannier90 as a community code: new features and applications, J. Phys. Condens. Matter 32, 165902 (2020).

[61] G. Li et al., Surface states in bulk single crystal of topological semimetal $\mathrm{Co}_{3} \mathrm{Sn}_{2} \mathrm{~S}_{2}$ toward water oxidation, Sci. Adv. 5, eaaw9867 (2019).

[62] S.-M. Huang et al., A Weyl Fermion semimetal with surface Fermi arcs in the transition metal monopnictide TaAs class, Nat. Commun. 6, 7373 (2015). 